\title{
CAP: A ContAct based Proximity Service via Opportunistic Device-to-Device Relay
}

\author{
Tianhao Guo ${ }^{1}$, Gang Huang ${ }^{2}$, John Schormans ${ }^{1}$, Tong Wang ${ }^{3}$, and Yue $\mathrm{Cao}^{4}$ \\ ${ }^{1}$ School of Electronic Engineering and Computer Science Queen Mary University of London, London, UK \\ ${ }^{2}$ Reform Management Board Shanxi Transformation and Demonstration Zone, Taiyuan, China \\ ${ }^{3}$ College of Information and Communication Engineering, Harbin Engineering University, Harbin, China \\ ${ }^{4}$ Department of Computer and Information Sciences, Northumbria University, Newcastle upon Tyne, UK \\ ${ }^{1}$ Email: t.guo; j.schormans@qmul.ac.uk \\ ${ }^{2}$ Email: sxtyhg@21cn.com \\ ${ }^{3}$ Email: wangtong@hrbeu.edu.cn \\ ${ }^{4}$ Email: yue.cao@northumbria.ac.uk
}

\begin{abstract}
The research progress of $5 \mathrm{G}$ has brought a number of novel technologies to meet the multi-dimensional demands. Device-to-Device (D2D) communication is a way to no longer treat the User Equipments (UEs) as terminal, but rather as a part of network (known as helpers) for service provisioning. Such a way potentially increases the coverage and also expands the capacity of cellular network. In this paper, we propose a generic framework for Proximity as a Service (PaaS) with demands of service continuity, namely ContAct based Proximity (CAP) via opportunistic D2D communication. Mainly, fruitful contact information (e.g., contact duration, frequency and interval) is captured as a key metric, to handle an ubiquitous and PaaS through the optimal selection of helpers. The nature of CAP is evaluated under the Helsinki city scenario, with key factors influencing the service demands (e.g., success ratio, disruption duration and frequency). Simulation results show the advantage of CAP, in both success ratio and continuity of the service. This work is the first one to evaluate LTE-Direct and WiFi-Direct in opportunistic proximity services.
\end{abstract}

Index Terms-D2D, PaaS, Human Mobility, LTE-Direct, Opportunistic Communication.

\section{INTRODUCTION}

In recent years, the explosive growth of miscellaneous devices along with various service demands, has brought numerous challenges, e.g., growth of data traffic by orders of magnitude, ubiquitous coverage and unimaginable variety of service requirements to current network infrastructures. The needs to increase the network capacity and coverage, have been commonly recognized as a key feature for the Fifth Generation of mobile networks (5G). Several killer technologies, e.g., massive multiple-input-multiple-output (MIMO), millimeter wave (mmWave), non-orthogonal multiple access (NOMA) and heterogeneous networks (HetNets) have been extensively studied in the literature [1].

Alternatively, Device-to-Device (D2D) is a much cheaper solution [2], by enabling direct communication between mobile devices without necessarily involving cellular links in data transmissions. Thanks to the developing features of smart phones, User Equipments (UEs) with such powerful smart phone would be able to play an attractive role as service suppliers in proximity, other than as requesters in nature.

Proximity as a Service (PaaS) proposed by 3GPP Release12 [3] brings a promising market, via the D2D communication. Basic functions for D2D communication include discovery, one-to-one communication, one-to-many communication and UE relaying. Up-to-now, the exploration of use cases with different service requirements is in full swing, including cellular traffic offloading [4], opportunistic crowd computing [5], mobile augmented reality [6], mobile crowdsourcing [7], computation offloading [8], target marketing [9] and even block-chain applications [10] etc.

In this paper, we propose a new paradigm by which a number of (appropriately selected) UEs, namely helpers [4], are eligible to opportunistically provide PaaS for other UEs (as service requesters) in need. Key techniques here involve sophisticated design of signalling protocol, necessary knowledge awareness of human mobility, efficient D2D handling for specific services requirements, and potential security issues etc. Among these, we are mainly interested in following key issues:

- How to explore human mobility, so as to identify a certain number of appropriate helpers (in terms of whom to help) for PaaS? We formulate the helpers selection problem and propose the ContAct based Proximity (CAP), which is puns for "CAP" with the meaning of covering the demands of UEs ubiquitously (and ideally seamlessly). Fruitful contact history information such as contact duration, contact frequency and inter-meeting duration is captured for human mobility prediction.

- What are the key factors influencing the success ratio and continuity of PaaS? Here, the continuity refers to the least disruption (in terms of frequency and time duration) experienced during a service. Inevitably, D2D connections suffer disruption by nature [11], because of human mobility and limitation of device transmission range, e.g., WiFi-Direct is generally with 50 meters 


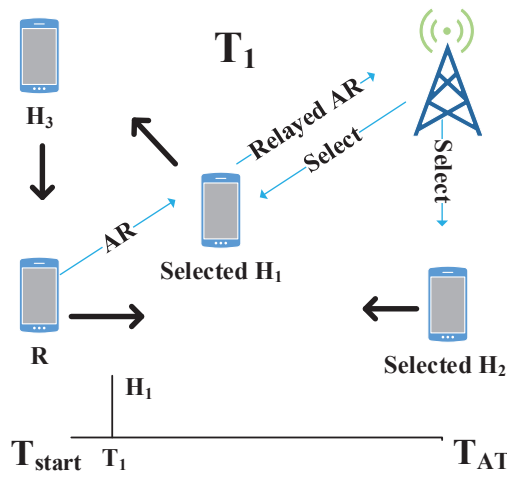

(a)

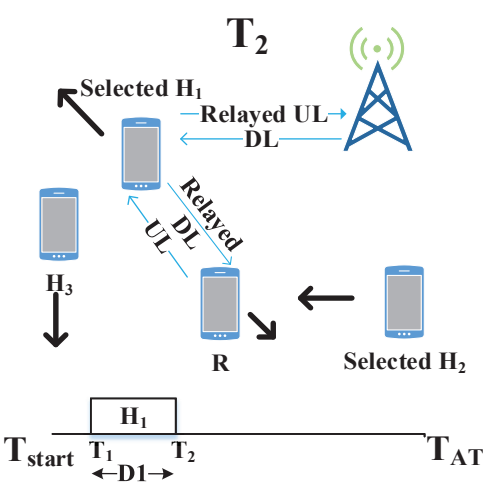

(b)

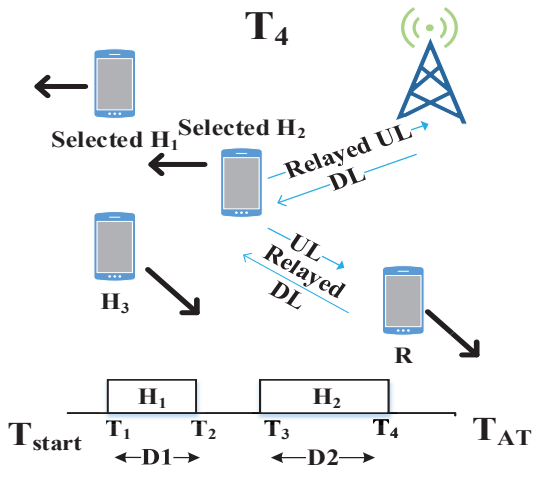

(c)

Fig. 1. Process of D2D Relaying

transmission range. According to our previous work [12], edge computing such as TCP proxy implemented in service providers can enable services in disturbed wireless environments. However, with an even more challenging condition, e.g., a 5 hours' disruption, the service would normally fall into the Delay/Disruption Tolerant Networking [4], [13], [14] paradigm.

There have been several techniques of D2D air interfaces, such as in-band underlay D2D, in-band dedicated D2D and out-band D2D [15]. In this paper, both out-band D2D communication and in-band dedicated D2D communication for UERelay are considered in PaaS.

Different D2D air interfaces represent different transmission range. It is worth noting that the Qualcomm Inc has always been the only rapporteur of specifications related to PaaS in 3GPP. The Qualcomm is also the first one to develop the chipset for in-band D2D LTE-Direct [16]. The maximal transmit range of LTE-Direct is designed to be $500 \mathrm{~m} \mathrm{[16],}$ because dedicated in-band D2D technique can use its own bands which are suitable for longer transmit range without interference. However, with the consideration of shadow loss, multi-path effects and sensitivity of battery life, the transmit range of LTE-Direct implemented in smart phones in urban areas cannot reach $500 \mathrm{~m}$.

\section{RELATED WORK}

The majority of previous works [17] on D2D focus on how to cognitively allocate spectrum or energy for paired UEs, in order to achieve a higher throughput with less interference. However, they fail to investigate the impact of human mobility on the network performance. Due to the opportunistic nature of D2D communication, long/frequent disruption or delay is inevitable, especially in a multi-hop case. Therein, the end-toend based multi-hop path from source to destination, cannot be established due to intermittent links. Fruitful efforts in the field of Delay Tolerant Network (DTN) [18] and mobile social networks (e.g., content offloading [4] and mobile crowdsourcing [7]), have proposed a number of ways to estimate the delivery potential of relay for improved message delivery.
However, in the 5G era, network facilities are almost ubiquitous wherever people live. As such, the majority of D2D communication may just rely on a few hops, known as PaaS. Inevitably, due to the selfish nature of human beings and potential security issues, not all the UEs are willing to act as helpers for PaaS. From the perspective of the operator, selecting the minimum number of helpers to provide satisfactory service is also cost-efficient. After all, the operator would prefer not to loose its control. Several PaaS have been proposed, mainly addressing how to achieve QoS awareness performance [6]-[10], through appropriate helpers selection. On the contrary, we propose the CAP to provide ubiquitous (high service success ratio) and QoE like (e.g., disruption duration and frequency) service, through extensive effort to explore human mobility and access handling run by helpers.

\section{SYSTEM MODEL}

We consider a system with a certain number of Base Stations (BSs), Cellular UEs (CUEs) and UEs that need to access the network by D2D communication. The CUEs are assumed to be willing to help other UEs access the network by D2D communication.

The CUEs that help other UEs as relay nodes are defined as helpers. UEs that need to access the network by D2D communication with helpers are defined as requesters. The helpers are acting as "mobile access points" or "mobile picocells" with wireless backhual links [19].

The whole communication process has two hops: the first hop is between the requester to the helper; the second hop is D2D communication between the helper to the BS. Helpers are assumed to have stable connections with infrastructure. Nevertheless, the opportunistic contact between pairwise helper and requester, inevitably results in the service disruption.

\section{A. An Example of CAP}

Here is an example of a CAP use case. We assume that there are three helpers, one requester and one base station in the CAP system. The requester is assumed to generate access requests with the same requested access duration $\left(T_{R A D}\right.$, e.g., half an hour). The access request needs to be served within a period of time, called the access tolerance ( $T_{A T}$, e.g.,2 hours). 
The time clip of the process of CAP in Fig. 1 begins at the start of an access request $\left(T_{\text {start }}=0\right)$ and finishes at the end of the access tolerance $\left(T_{A T}\right)$ of the access request. The moments $T_{1}, T_{2}$ and $T_{4}$ are shown to elaborate the CAP process.

- At $T_{1}$, an access request with $T_{R A D}$ and $T_{A T}$ was generated from a requester. The requester, running D2D air interfaces, broadcasts access request $(A R)$ to nearby devices periodically through an associated mobile application. As illustrated in Fig. 1(a), when helper $1\left(H_{1}\right)$ received the $A R$ and relayed the $A R$ to the base station. The base station then arranged and selected appropriate helpers $\left(H_{1}\right.$ and $\left.H_{2}\right)$ for this requester based on metrics (utilities) derived from their contact history.

- At $T_{2}$, all the UEs had moved to different locations, as illustrated in Fig. 1(b). $H_{1}$ had helped the requester $(R)$ access the network for a period of duration $D_{1}\left(D_{1}<\right.$ $\left.T_{R A D}\right)$. However, $H_{1}$ was going to leave $R$.

- In Fig. 1(c) taken at $T_{4}, H_{2}$ began to help $R$ at $T_{3}$ and finished the service at $T_{4}\left(D_{1}+D_{2}=T_{R A D}\right)$, which is within $T_{A T}$.

The system is assumed to be based on one-to-many D2D, which means that a helper can have connections with several requesters but a requester can only communicate with one helper.

TABLE I

List of NOTATIONS FOR PRoblem Formulation

\begin{tabular}{|l|l|}
\hline$T_{A T}$ & Access Tolerance \\
\hline$T_{R A D}$ & Requested Access Duration \\
\hline$R_{j}$ & Requester $\mathrm{j}$ \\
\hline$H_{i}$ & Helper $\mathrm{i}$ \\
\hline$N_{H}$ & Total number of helpers in the system \\
\hline$N_{R}$ & Total number of requesters in the system \\
\hline$A R_{j k}$ & The $\mathrm{k}$ th access request of requester $\mathrm{j}$ \\
\hline$T_{A R_{j k}}$ & Start time of the $\mathrm{k}$ th access request of requester $\mathrm{j}$ \\
\hline$S H_{A R_{j k}}$ & Set of selected helper for $A R_{j k}$ \\
\hline$M$ & limit number of the set $S H_{A R_{j k}}$ \\
\hline$S_{A R}$ & Total number of all the generated access requests \\
\hline$S C_{A R}$ & Total number of the completed access requests \\
\hline$K$ & Total number of requester a help can serve at the same time \\
\hline$T_{j k}^{\text {ela }}$ & Elapsed time since $A R_{j k}$ requested \\
\hline
\end{tabular}

\section{B. Problem Formulation}

It is true that not all helpers will be allocated with the role to provide access service to requesters. Therefore, how to optimally select a certain number of helpers will be of interest. An accurate and concise mathematical description of the helper selection problem is the first step.

Equation (1) shows that at any time, the contact relationship between $H_{i}$ and $R_{j}$ can be contacted or not contacted.

$$
H_{i}(t) R_{j}(t)= \begin{cases}1 & \text { if contacted } \\ 0 & \text { otherwise }\end{cases}
$$

When base stations receive an access request $A R_{j k}$, a optimal set of helpers (e.g., in terms of minimized helpers number) is selected as $S H_{A R_{j k}}$ to serve this $A R_{j k}$. If $H_{i}$ is in the selected set of helpers $S H_{A R_{j k}}$, the accumulated contact duration between $H_{i}$ and $R_{j}$ as denoted by $T\left(H_{i} A R_{j k}\right)$, within $T_{A T}$ of $A R_{j k}$, is shown in Equation (2).

$$
T\left(H_{i} A R_{j k}\right)=\int_{T_{A R_{j k}}}^{T_{A T}+T_{A R_{j k}}} H_{i}(t) R_{j}(t) d t .
$$

The results of the service contributed by the selected helpers $\left(S H_{A R_{j k}}\right)$ are: completed or not completed. As shown in Equation (3), the binary value " 1 " represents completed, which means that the sum of accumulated contact duration between the selected helpers and the requester $R_{j}$ is not less than $T_{R A D}$, while the binary value " 0 " represents not completed.

$$
C\left(A R_{j k}\right)= \begin{cases} & \text { if } \sum_{i}^{S H_{A R}} T\left(H_{i} A R_{j k}\right) \geqslant T_{R A D} \\ 0 & \text { otherwise }\end{cases}
$$

The helper selection strategy can be formulated as an optimization problem. It consists of the objective function (maximizing total number of the completed access requests $S C_{A R}$ ), by choosing subsets (selected helpers $S H_{A R_{j k}}$ ) for all access requests (with the total number $S_{A R}$ ), from all helpers in the system. This problem is subjected to two limitations. Firstly, a helper can only serve limited requesters simultaneously; secondly, the number of helpers serving a requester is limited. In other words, the cardinality of the set $S H_{A R_{j k}}$, namely $\operatorname{Card}\left(S H_{A R_{j k}}\right)$, is limited.

$$
\begin{array}{ll}
\max & S C_{A R}=\sum_{i=1}^{S_{A R}} C\left(A R_{j k}\right) \\
\text { s.t. } & \sum_{j=1}^{N_{R}} H_{i}(t) R_{j}(t) \leqslant K, \quad i=1,2,3 \ldots, N_{H} \\
& \operatorname{Card}\left(S H_{A R_{j k}}\right) \leqslant M, \quad j=1,2,3 \ldots, N_{R}
\end{array}
$$

\section{Discussion on the System}

The mobility of the UEs is not prior knowledge, so the information of Equation (1) is unknown before the experiment. Without this information, it is hard to estimate the results of the access requests. For example, $A R_{j 1}$ denotes the first access request from $R_{j}$. If $A R_{j 1}$ is completed, it is also hard to estimate the service completed time. A requester can only have one access request at the same time, which means that the $A R_{j 2}$ can only be generated after the finishing time of $A R_{j 1}$. In other words, the more access requests completed, the more access requests generated. So the total number of access requests $\left(S_{A R}\right)$ is also unknown.

What's more, when $R_{j}$ is temporarily isolated, $A R_{j k}$ from $R_{j}$ is hard to be completed, even if all the encountered helpers serve it, as shown in Equation (5). This situation is also unpredictable.

$$
\sum_{i=1}^{N_{H}} T\left(H_{i} A R_{j k}\right)<T_{R A D} .
$$

Based on the difficulties brought by the unpredictable mobility of the nodes, this optimization problem is hard to solve. Therefore a heuristic algorithm which can learn the information of contact history is a feasible approach. The helper can also raise the priority of requesters based on elapsed time $T_{j k}^{e l a}$ if the number of concurrent devices is more than $K$. 


\section{The Algorithm for Helper Selection}

TABLE II

LIST OF NOTATIONS FOR ALGORITHM

\begin{tabular}{|l|l|}
\hline$D_{i, j}$ & Historically contact duration between $H_{i}$ and $R_{j}$ \\
\hline$T_{i, j}$ & Historically inter-meeting time between $H_{i}$ and $R_{j}$ \\
\hline$C_{i, j}$ & Historically contact count between $H_{i}$ and $R_{j}$ \\
\hline$U_{i, j}$ & Utility value estimated of $H_{i}$ and $R_{j}$ \\
\hline$U A_{i, j}$ & Utility for helper selection, $A$ means average \\
\hline$\Gamma$ & aging constant \\
\hline$N_{i, j}$ & Total number of contacts between $H_{i}$ and $R_{j}$ \\
\hline
\end{tabular}

The idea of this algorithm is learning the regularity of mobility from contact history, and then, proposing a utility for every helper and requester pair. The utilities are the scores to rank the appropriate helpers for a requester.

\section{A. Utility Function Design}

The contact history between a helper and a requester is elaborated in Fig. 2. $T_{i, j}^{\left(C_{i, j}=1\right)}$ means the moment when $H_{i}$ and $R_{j}$ contact for the first time. The inter meeting time shown in Fig. 2 is $T_{i, j}^{\left(C_{i, j}=2\right)}$. For $C_{i, j} \geqslant 2, T_{i, j}^{\left(C_{i, j}\right)}-D_{i, j}^{\left(C_{i, j}-1\right)}$ means the encounter gap, namely disruption duration. Note that the $C_{i, j}$ can only be captured upon the new contact happens.

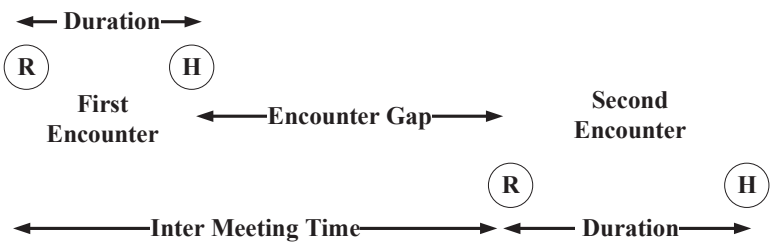

Fig. 2. Illustration of Contact History

Based on the information obtained from contact history between a pairwise helper and requester, the regularity can be found. For example, there are one requester $(R)$ and two helpers $\left(H_{1}\right.$ and $\left.H_{2}\right)$ in the system. $H_{1}$ contacts with $R$ frequently, with longer contact duration while with less disruption duration. $H_{2}$ rarely contacts $R$, with shorter contact duration while with longer disruption duration. It is easy to learn that $H_{1}$ will be a better choice for $R$. Moreover, $T_{H_{i}, R_{j}}^{\left(C_{i, j}=1\right)}$ also implies how fast $R_{j}$ can get connected to network. Based on this observation, we can obtain an empirical utility function, as shown in Equation (6).

$$
U_{i, j}=\frac{T_{i, j}^{\left(C_{i, j}=1\right)}+\sum_{i, j}^{N_{i, j}}\left(C_{i, j}=2\right)\left(T_{i, j}^{\left(C_{i, j}\right)}-D_{i, j}^{\left(C_{i, j}-1\right)}\right)}{N_{i, j}}
$$

Take the above as an example, there is one requester $\left(R_{1}\right)$ and two helpers $\left(H_{1}\right.$ and $\left.H_{2}\right)$ in the system. Assuming $T_{1,1}^{\left(C_{1,1}=1\right)}=15, D_{1,1}^{\left(C_{1,1}=1\right)}=3$ at the first contact, $T_{1,1}^{\left(C_{1,1}=2\right)}=10$ and $D_{1,1}^{\left(C_{1,1}=2\right)}=6$ at the second contact, while $T_{1,1}^{\left(C_{1,1}=3\right)}=20$ is recorded at the third contact, then $U_{1,1}$ is calculated as:

$$
U_{1,1}=\frac{15+(10-3)+(20-6)}{3}=12
$$

Assuming $T_{2,1}^{\left(C_{2,1}=1\right)}=20, D_{2,1}^{\left(C_{2,1}=1\right)}=2$ at the first contact, while $T_{2,1}^{\left(C_{1,1}=2\right)}=20$ is recorded at the third contact, then $U_{2,1}$ is calculated as:

$$
U_{2,1}=\frac{20+(20-2)}{2}=19
$$

It is obvious that a helper with a smaller value of utility has the potential to provide good quality of service, in terms of success ratio and continuity. It is also worth mentioning that the utility is updated at the beginning of the contact. Contact duration is recorded when the contact is disrupted, while intermeeting is recorded at the beginning of the contact, similar to $C_{i, j}$, as shown in Algorithm 1.

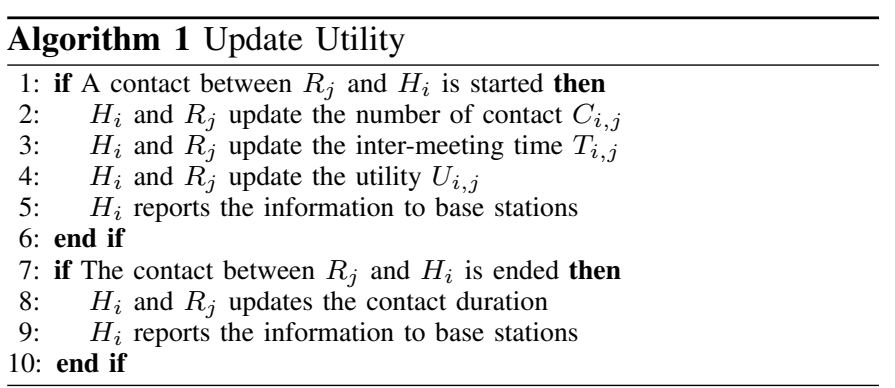

The relationship between nodes is relatively steady while the contact may fluctuate, so $U_{i, j}$ is not eligible to accurately reflect the proximity (in terms of how often and how continuous) between $\mathrm{i}$ and $\mathrm{j}$. We use $U A_{i, j}$ as the utility to make helper selection. $U A_{i, j}$ is updated by Exponential Moving Average (EMA) by every connection, as shown in Equation (9), where $\Gamma$ is the weighted constant.

$$
U A_{i, j}{ }^{(\text {new })}=U A_{i, j}{ }^{(\text {previous })} \times \Gamma+U_{i, j} \times(1-\Gamma)
$$

\section{B. Access Handling}

When base stations receive an access request from a requester relayed by a helper, all the helpers would be sorted based on utilities and the best $M$ helpers would be selected $\left(S H_{A R_{j k}}\right)$.

A helper can only serve a limited number $(K)$ of requesters simultaneously. If base stations arrange too many devices for this helper, the most urgent requesters would be chosen to serve based on $T_{j k}^{e l a}$.

\section{Performance Evaluation}

Here the evaluation is based on the medium Helsinki city scenario in Opportunistic Network Emulator (ONE) [20], as shown in Fig. 4. We deploy 4 types of interests points, namely Point Of Interest (POI), on this map with the consideration of the regularity of movement. For example, nodes in area- 4 would randomly move to one of the 22 points by map-based shortest path movement pattern (Dijkstra's algorithm).

As shown in TABLE III, there are four groups of requesters and 4 groups of helpers which have different probabilities of being in four areas, namely mobility patterns. The requesters of the first group (R1) spend the majority of time in Area-1 while requesters in group 2 (R2) and group 3 (R3) spend more 


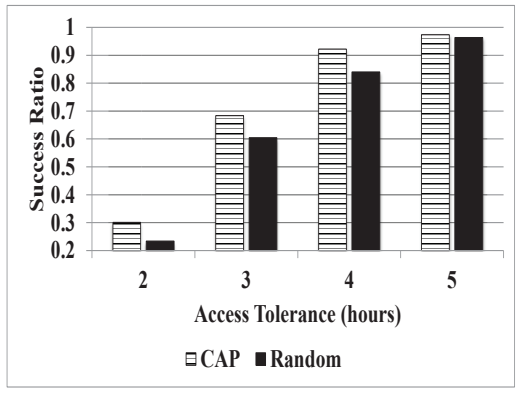

(a) Success Ratio

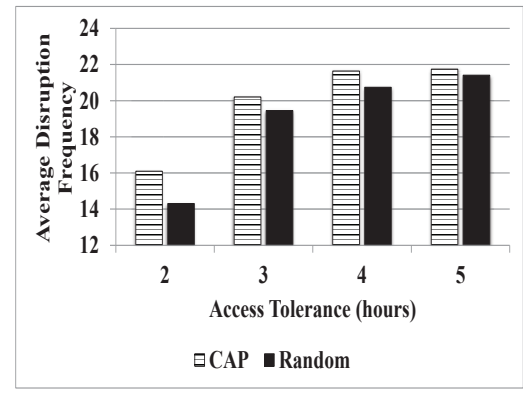

(b) Average Disruption Frequency

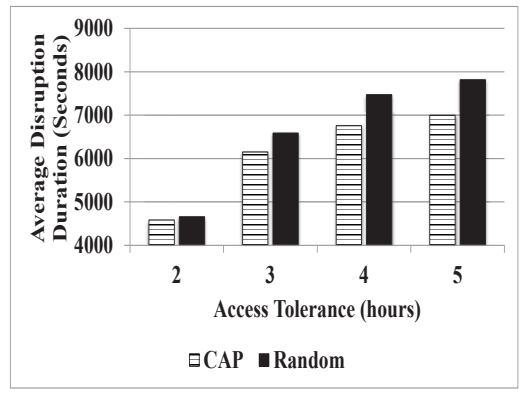

(c) Average Disruption Duration

Fig. 3. Influence of Access Tolerance

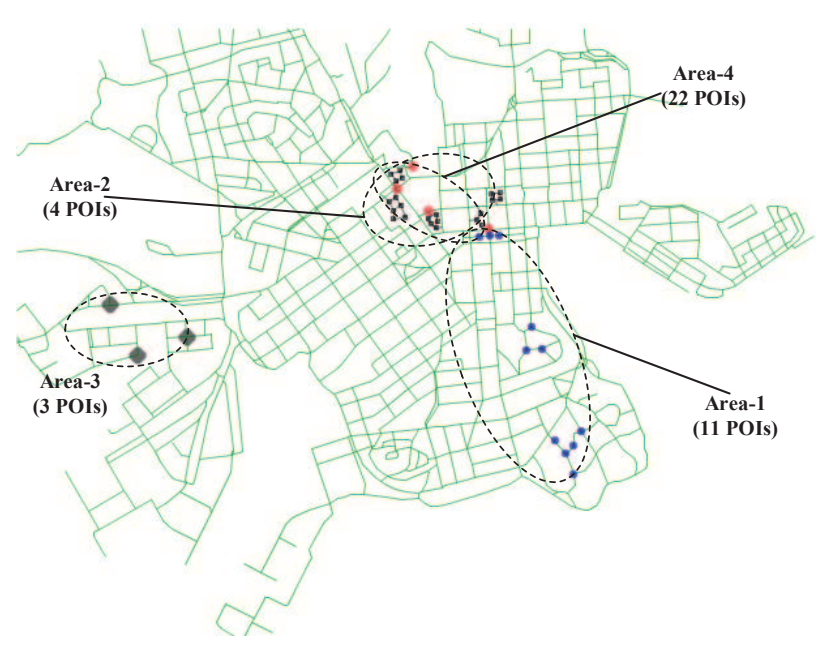

Fig. 4. Medium Helsinki City Scenario with POI

time in Area-2 and Area-3 respectively. Requesters in group 4 (R4) spend most of time to move in Area-4 compared to requesters in other groups. Similarly, one hundred helpers also move within 4 POIs with four mobility patterns.

TABLE III

Probabilities of Movement in Four Areas

\begin{tabular}{|l|l|l|l|l|l|}
\hline Group & Number & Area-1 & Area-2 & Area-3 & Area-4 \\
\hline R1 & 25 & 0.7 & 0.1 & 0.1 & 0.1 \\
\hline R2 & 25 & 0.1 & 0.7 & 0.1 & 0.1 \\
\hline R3 & 25 & 0.1 & 0.1 & 0.7 & 0.1 \\
\hline R4 & 25 & 0.1 & 0.1 & 0.1 & 0.7 \\
\hline H1 & 25 & 0.7 & 0.1 & 0.1 & 0.1 \\
\hline H2 & 25 & 0.1 & 0.7 & 0.1 & 0.1 \\
\hline H3 & 25 & 0.1 & 0.1 & 0.7 & 0.1 \\
\hline H4 & 25 & 0.1 & 0.1 & 0.1 & 0.7 \\
\hline
\end{tabular}

The D2D air interface is configured as WiFi-Direct (outband D2D [15]), with $50 \mathrm{~m}$ range. We assume all the users in the system are moving with speed varies between $[0.5 \sim 1.5]$ $\mathrm{m} / \mathrm{s}$. The one hundred requesters generate network access requests after $10800 \mathrm{~s}$ warm-up time, so the regularity of the mobility can be learned by base stations after a 3 hours movement. Without loss of generality, the requesters are assumed busy, which means they generate new requests with an interval of $60 \mathrm{~s}$ after successful access service or after $T_{A T}$
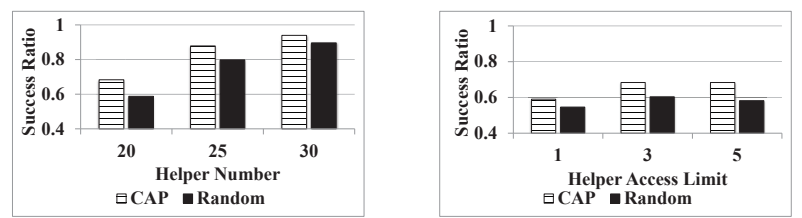

Fig. 5. Influenced by Helper Number Fig. 6. Influenced by Access Limit

(access tolerance). All the requests are assumed to require $1800 \mathrm{~s}$ (half an hour) of access duration. The simulation time is set as $57600 \mathrm{~s}$ (16 hours).

Here we compare our helper selection algorithm CAP with the "Random" (which means that the $M$ helpers serving requesters are randomly selected from 100 helpers). The algorithm is evaluated by $T_{A T}$ (access tolerance) on:

- Success Ratio: The percentage of completed service.

- Average Disruption Frequency: The average number of disruptions during each access service.

- Average Disruption Duration: The average duration of each access request.

We also evaluate the success ratio by the influence of $M$ (the number of selected helpers) and $K$ (helper access limit).

\section{A. Influence of Access Tolerance}

The access tolerance $T_{A T}$ is set as 2 hours, 3 hours, 4 hours and 5 hours respectively, with transmit range of $50 \mathrm{~m}, 20$ selected helpers $(M=20)$ and access limit of 5 concurrent requesters $(K=5)$. As shown in Fig. 3(a), the success ratio of CAP increases rapidly, along with the growth of access tolerance, from $30 \%$ in 2 hours, $68 \%$ in 3 hours, $92 \%$ in 4 hours to $97 \%$ in 5 hours. The CAP outperforms Random, by about $7 \%$ in 2, 3 and 4 hours. However, when it comes to 5 hours, Random can also reach $96 \%$. In terms of average disruption frequency, CAP and Random both increase side by side with the length of access tolerance. CAP outperforms Random in average disruption duration and both of them grow with access tolerance, because larger access tolerance can tolerate longer disruption duration.

\section{B. Influence of Selected Helper Number and Helper Access Limit}

Herein the number of selectable helpers $M$ is set as 20, 25,30 respectively, with transmit range of $50 \mathrm{~m}, 3$ hours' 
access tolerance $\left(T_{A T}=3\right)$ and access limit of 5 concurrent requesters $(K=5)$. As shown in Fig. 5 , the success ratio is sensitive to $M$, with steps of 5 leading to a soar from $68 \%$ to $88 \%$ and finally $93 \%$ by CAP. CAP outperforms Random in success ratio since appropriately selected helpers are able to successfully relay requesters. In Fig. 6 , access limit $K$ is set as $1,3,5$ respectively, with $M=20$, transmit range of 50 $\mathrm{m}$ and $T_{A T}=3$. CAP also outperforms Random in success ratio. $K=3$ outperforms $K=1$ but is the same with $K=5$, which means that there are rarely five concurrent requesters connected to one helper.

\section{Influence of Transmit Range (LTE-Direct)}

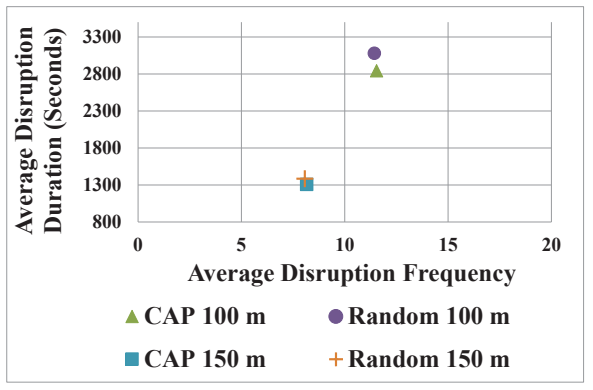

Fig. 7. Influence of Transmit Range

The transmit range of LTE-Direct is set as $100 \mathrm{~m}$ and $150 \mathrm{~m}$, with 20 selected helpers $(M=20), 3$ hours' access tolerance $\left(T_{A T}=3\right)$ and access limit of 5 concurrent requesters $(K=5)$. Only continuity of PaaS is shown in Fig. 7 since success ratio is always $100 \%$ in both cases. Transmit range influences the duration of every contact between pairwise helper and requester, so the average number of disruptions (contacts) during each access service is the same in CAP and Random if success ratio is $100 \%$. When transmit range is 150 $\mathrm{m}, \mathrm{CAP}$ and Random can both work well. Such an observation implies that miscellaneous helpers with longer transmit ranges can provide seamless PaaS. For example, drones implemented with in-band D2D air interfaces suffer less shadow loss, multipath effects and sensitivity of battery life compared to smart phones.

\section{CONCLUSION}

In this paper, we formulate and propose a heuristic scheme CAP, to facilitate the selection of appropriate helpers for PaaS. Simulation results show the advantage of our proposed CAP, in both success ratio and continuity of the service. Results further reveal the influence of four factors, e.g., service tolerance, number of helpers allocated, the number of concurrent devices supported by each helper and transmit range, on the PaaS through opportunistic D2D relay.

The future work will introduce miscellaneous devices with increasing number of selectable helpers and various transmit ranges to get the ubiquitous and seamless PaaS. The battery consumption and bandwidth will be added in the experiments.

\section{REFERENCES}

[1] A. Gupta and R. K. Jha, "A survey of $5 \mathrm{~g}$ network: Architecture and emerging technologies," IEEE access, vol. 3, pp. 1206-1232, 2015.

[2] K. Doppler, M. Rinne, C. Wijting, C. B. Ribeiro, and K. Hugl, "Deviceto-device communication as an underlay to lte-advanced networks," IEEE Communications Magazine, vol. 47, no. 12, pp. 42-49, Dec 2009.

[3] http://www.3gpp.org/specifications/releases.

[4] B. Han, P. Hui, V. Kumar, M. V. Marathe, G. Pei, and A. Srinivasan, "Cellular traffic offloading through opportunistic communications: a case study," in Proceedings of the 5th ACM workshop on Challenged networks. ACM, 2010, pp. 31-38.

[5] D. Chatzopoulos, M. Ahmadi, S. Kosta, and P. Hui, "Openrp: a reputation middleware for opportunistic crowd computing," IEEE Communications Magazine, vol. 54, no. 7, pp. 115-121, July 2016.

[6] D. Chatzopoulos and P. Hui, "Readme: A real-time recommendation system for mobile augmented reality ecosystems," in Proceedings of the 2016 ACM on Multimedia Conference, ser. MM '16. New York, NY, USA: ACM, 2016, pp. 312-316. [Online]. Available: http://doi.acm.org/10.1145/2964284.2967233

[7] C. Bermejo, D. Chatzopoulos, and P. Hui, "How sustainable is social based mobile crowdsensing? an experimental study," in 2016 IEEE 24th International Conference on Network Protocols (ICNP), Nov 2016, pp. $1-6$.

[8] K. Sucipto, D. Chatzopoulos, S. Kosta, and P. Hui, "Keep your nice friends close, but your rich friends closer - computation offloading using NFC," CoRR, vol. abs/1612.03000, 2016. [Online]. Available: http://arxiv.org/abs/1612.03000

[9] N. Allurwar, B. Nawale, and S. Patel, "Beacon for proximity target marketing," Int. J. Eng. Comput. Sci, vol. 15, pp. 16359-16364, 2016.

[10] D. Chatzopoulos, S. Gujar, B. Faltings, and P. Hui, "Localcoin: An ad-hoc payment scheme for areas with high connectivity: Poster," in Proceedings of the 17th ACM International Symposium on Mobile Ad Hoc Networking and Computing, ser. MobiHoc '16. New York, NY, USA: ACM, 2016, pp. 365-366. [Online]. Available: http://doi.acm.org/10.1145/2942358.2947401

[11] S. Andreev, J. Hosek, T. Olsson, K. Johnsson, A. Pyattaev, A. Ometov, E. Olshannikova, M. Gerasimenko, P. Masek, Y. Koucheryavy, and T. Mikkonen, "A unifying perspective on proximity-based cellularassisted mobile social networking," IEEE Communications Magazine, vol. 54, no. 4, pp. 108-116, April 2016.

[12] Z. Jian, G. Tianhao, L. Jilong, and Y. Mingwu, "Adaptive flow control proxy for enhancing downlink tcp throughput in infrastructure wlans," in Communications and Information Technologies (ISCIT), 2014 14th International Symposium on. IEEE, 2014, pp. 56-60.

[13] N. Wisitpongphan, F. Bai, P. Mudalige, V. Sadekar, and O. Tonguz, "Routing in sparse vehicular ad hoc wireless networks," IEEE journal on Selected Areas in Communications, vol. 25, no. 8, 2007.

[14] Y. Cao, N. Wang, Z. Sun, and H. Cruickshank, "A reliable and efficient encounter-based routing framework for delay/disruption tolerant networks," IEEE Sensors Journal, vol. 15, no. 7, pp. 4004-4018, 2015.

[15] X. Lin, J. G. Andrews, and A. Ghosh, "A comprehensive framework for device-to-device communications in cellular networks," CoRR, vol. abs/1305.4219, 2013. [Online]. Available: http://arxiv.org/abs/1305.4219

[16] I. Qualcomm Technologies, "Lte direct always-on device-todevice proximal discovery," 2014.

[17] A. Asadi, Q. Wang, and V. Mancuso, "A survey on device-to-device communication in cellular networks," IEEE Communications Surveys \& Tutorials, vol. 16, no. 4, pp. 1801-1819, 2014.

[18] Y. Cao and Z. Sun, "Routing in delay/disruption tolerant networks: A taxonomy, survey and challenges," IEEE Communications Surveys Tutorials, vol. 15, no. 2, pp. 654-677, Second 2013.

[19] N. Golrezaei, K. Shanmugam, A. G. Dimakis, A. F. Molisch, and G. Caire, "Femtocaching: Wireless video content delivery through distributed caching helpers," in INFOCOM, 2012 Proceedings IEEE. IEEE, 2012, pp. 1107-1115.

[20] A. Keränen, J. Ott, and T. Kärkkäinen, "The one simulator for dtn protocol evaluation," in Proceedings of the 2nd international conference on simulation tools and techniques. ICST (Institute for Computer Sciences, Social-Informatics and Telecommunications Engineering), 2009, p. 55. 